\title{
ANTIFUNGAL SUSCEPTIBILITY OF BIOFILM PRODUCING Candida albicans ISOLATED FROM ORAL CAVITY OF DIABETIC AND NON-DIABETIC POPULATION OF DHARAN, NEPAL
}

Bijay kumar Shrestha ( $\nabla$ interfacebj@gmail.com )

Tribhuvan University Institute of Science and Technology https://orcid.org/0000-0002-6542-829X Jenish Shakya

Central Campus of Technology,TU

Hemanta Khanal

Central Campus of Technology,TU

Research article

Keywords: Candida, Biofilm, Diabetes mellitus, Oral candidiasis, Drug Resistance

Posted Date: August 2nd, 2019

DOl: https://doi.org/10.21203/rs.2.12328/v1

License: (c) (i) This work is licensed under a Creative Commons Attribution 4.0 International License.

Read Full License 


\section{Abstract}

Background: Candida are almost universal on normal adult skin and $\mathrm{C}$. albicans is part of the normal flora of the mucous membranes of the respiratory, gastrointestinal, and female genital tracts. It is acknowledged that diabetic patients are more susceptible to infections caused by Candida albicans due to increased blood glucose and inability of immune system in eradicating the fungus. Studies suggest that Gutkha consumers are also at high risk of oral Candida carriage . Materials and methodology The participants were provided $10 \mathrm{ml}$ of Normal saline and were asked to oral rinse for 1 minute. Oral rinse was collected in a sterile screw capped container and was transported in cold chain to microbiology laboratory. The oral rinse sample was inoculated onto the Sabouraud dextrose agar with Chloramphenicol and was incubated at $37^{\circ} \mathrm{C}$ for 3-4 days. The Colony forming Unit of candida was compared among diabetic and healthy controls. The candida albicans were identified by Germ tube formation. The Candida albicans isolates were subjected to Biofilm assay, Antifungal susceptibility Test, Haemolysin assay, Haemolysis degree and phospholipase assay. Result: This study reported 31.5\% prevalence of oral Candida.The Candida carriage in CFU of diabetic population was statistically significant $(p<0.05)$. The maximum isolates were found to be Biofilm producers. There was significant association between Gutkha consumers with oral Candida carriage. The study suggests that there is higher colonization of Candida in diabetic populations than in healthy population. The result also concludes that frequency of Candida in Oral cavity of Gutkha consumers was also higher $(p<0.05)$. All isolated strains of Candida albicans were tested for antifungal susceptibility testing and $76.19 \%$ were found to be Resistant to Fluconazole and $50 \%$ were found to be resistant to Amphotericin B. There was statistical significance in Biofilm formation and fluconazole Drug resistance. Conclusion The findings indicated highest colonization of oral Candida in diabetic population and in Gutkha consumers. The greatest numbers of isolated Candida albicans were biofilm producer which showed greater frequency of Fluconazole drug resistance.

\section{Background}

Candida albicans is known to be normal flora of human body harboring skin, mucosal cavity oral, vaginal mucosa and known to colonize $60 \%$ of healthy population [1]. Candida albicans is dimorphic fungi isolated most commonly from Biofilms of medical devises and human tissue [2]. Studies have suggested that Candida albicans are associated with number of opportunistic infections in immunocompromised patients like in HIV patients, cancer patients and diabetic patients etc. [3].

In diabetes mellitus type-II there has been known greater incidence of oral candidiasis which could be associated with immune dysfunction caused by high glucose concentration in blood, tissue and saliva [4]. Individuals with diabetes were found to have higher Candida carriage rate than the non-diabetics, presumably due to increased Candida growth with high glucose levels in saliva, blood and neutrophil dysfunction [5]. 
Candida has known to be opportunistic pathogen under immunosuppression and tobacco chewing conditions [6, 7]. It is suggested that individuals chewing Gutkha are susceptible to oral Candida infections than non-chewers [8] .The greater prevalence and colonization of Candida in Gutkha consumers could be due to the presence of nicotine and hydrocarbons such as polycyclic aromatic hydrocarbons as a nutritional source for Oral yeast facilitating its growth [7.8]. Chewing Tobacco that includes Gutkha, Betel quid (BQ) which is common habit in South Asian nations like in India, Pakistan, Bangladesh, Sri lanka and Nepal [9]. Candida albicans pathogenesis is explained by its host defense mechanism, adherence, and production of tissue degrading hydrolytic enzymes like protease, phospholipase and haemolysin and role in biofilm production on host tissue and in medical devices [10]. Biofilm production is associated with the role of fungi to evade host immune function and overcome adhesions to host cell molecules to resist antifungal therapy making the treatment of infection more complicated [11].

Candida albicans exhibits hemolytic activity when grown on glucose-enriched blood agar [12]. In humans, most of the iron is located intracellular as ferritin or as heme-containing compounds. It is concluded that C. albicans expresses a hemolytic factor which allows it to acquire iron from host erythrocytes [13].

\section{Materials And Methods}

Research design: The cross sectional lab based study was carried out in microbiology laboratory of Central campus of Technology, Hattisar from June 2018 to November 2018. During the study 200 oral rinse samples were analyzed. All the work concerning this research was carried out in microbiology laboratory in Central Campus of Technology, TU, Hattisar, Dharan. The different oral rinse samples analyzed were from different potential risk population (50-barbers, 50-municipal waste workers, 50diabetic and 50-healthy individuals).Ethical approval was obtained from Nepal Health Research Council, Kathmandu. The research participants were informed about the procedure of sample collection and the informed consent was obtained in written form. All the participants without oral lesions were included in the study. The Healthy population was selected by Nutritionist on basis of physical fitness, good oral hygiene whereas Diabetic population was selected on basis of blood glucose level obtained from their medical history. All the socio-demographic information like brushing habit, Gutkha chewing e.t.c was obtained from research participants through Schedule.

\section{Inclusion criteria}

Group I patients: In the inclusion criteria, 50- participant having DM without any other oral lesions with following criteria were included in the study. Participants who have not received antibiotic and corticosteroid therapy before 4 weeks were included in study.

1. Random blood sugar (RBS) $\geq 200 \mathrm{mg} / \mathrm{dl}$ or

2. Fasting blood sugar (FBS) $>126 \mathrm{mg} / \mathrm{dl}$. 
Group II (Control group): 50-People who did not have DM or any other systemic illness, who were nutritionally fit, without any clinical signs of diseases, without any clinical medication were included as Healthy control.

Group III: It included 50-Barber population across the city.

Group IV: It included 50-Municipal waste worker of Dharan Sub metropolitan city.

\section{Exclusion criteria}

The people who never met above criteria were included under exclusion criteria

\section{Sample collection}

Ten $\mathrm{ml}$ of sterile saline were allowed to be rinsed for 1 minute and inoculated in a broader capped sterile container. The oral rinse sample for study were collected from the sample population and transported to microbiology laboratory maintaining cold chain .All the collected samples were labeled with participant's identification number. In case of delay, the sample was usually stored at $4^{\circ} \mathrm{C}$ in the refrigerator.

\section{Processing of oral rinse in SDA}

An aliquot of 50 microliter of oral rinse sample were inoculated in SDA (HiMedia, Mumbai, India) with chloramphenicol $(0.05 \mathrm{~g} / \mathrm{l})$ and incubated at $37^{\circ} \mathrm{C}$ for $24-48$ hours. Pure culture was identified by colony characteristics, grams staining. The number of colony was counted by Colony Counter and expressed as $\mathrm{CFU} / \mathrm{ml}[14,15]$.

\section{Assessment of Candida colonization by Quantitative Enumeration}

Number of colonies formed after incubation were counted by colony counter counted and multiplied with a factor of 20 to get the colonies in $1 \mathrm{ml}$ of a subject's sample [15].

Number of colonies contained in $50 \mu$ of sample $=n$

Therefore the number of colonies in $1000 \mu \mathrm{l}$

$=\mathrm{n} \times 1000 / 50$

$=\mathrm{n} \times 20$

\section{Identification of Candida albicans}


Germ tube and Chlamydospore formation was evaluated as described by Beheshti et al (1975) [16]. Germ tube test: The pure isolated colony of candida albicans was dispensed in $0.5 \mathrm{ml}$ of serum and incubated at $37^{\circ} \mathrm{C}$ for 2 hours. After incubation the aliquote was taken in a clean slide and was observed under microscope for the formation of Germ-tube.

Chlamydospore formation test: The pure isolated colony of Candida that could form Chlamydospore in Corn Agar was identified as Candida albicans.

\section{Hemolysin Assay}

Haemolysin activity was evaluated according to Luo et al 2001[13]. Hemolysin production by Candida albicans was performed by inoculation overnight culture of yeast on Sugar-enriched sheep blood agar as described by Manns et al (1994) [13]. The Blood base agar media was prepared by adding 5-7 ml of fresh blood to Saboured Glucose Agar with $3 \%$ glucose. $10 \mu \mathrm{L}$ of the yeast inoculum was placed at the centre of the plates. The plates were incubated at $37^{\circ} \mathrm{C}$ in $5 \% \mathrm{CO}_{2}$ for 48 hours. Hemolytic Index (Hz value) which represents the Hemolysin production is the ratio calculated by dividing the total diameter of the colony plus the translucent halo by the diameter of the colony.

\section{Screening Candida albicans for production of phospholipase}

The phospholipase test was done according to Samranayakae et al (1984) [17]. Egg yolk agar media was inoculated by $2 \mu \mathrm{L}$ of the inoculum and allowed to dry at room temperature. The plates will be incubated at $37^{\circ} \mathrm{C}$ for 3-4 days. $P z$ will be measured by dividing the diameter of the colony by the sum of diameter of the colony and the zone.

\section{Screening impact of diabetes in hemolytic activity}

The hemolytic activities were tested on Saboured dextrose Broth liquid media (SDB) containing 7\% defibrinated human blood according to Malcok et al (2009) [12]. One was supplemented with $3 \%$ glucose and the other without glucose. Candida albicans culture was inoculated and incubated for $48 \mathrm{hrs}$. The hemolysis in the media was detected spectrophotometrically by measuring the amount of released hemoglobin and compared with a standard hemolysate which was prepared prior to testing. The degree of hemolysis (percentage value) by an individual strain was calculated according to the following formula below: (Absorbance of supernatant media at $540 \mathrm{~nm} /$ Absorbance of standard hemolysate at $540 \mathrm{~nm} \times 100)$.

\section{Biofilm Assays}

\section{Microtitre plate method}


The quantification of biofilm was performed according to Christensen et al (1985) [18]. In this method, 5 $\mathrm{ml}$ of overnight culture of Candida albicans was prepared. Then 100 microliter of diluted culture was inoculated in a sterile 96-well polystyrene well plates well containing TSB with glucose. The plate was incubated at $37^{\circ} \mathrm{C}$ for 24 hours for biofilm production. The unbound cell was discarded and washes several times. $125 \mu \mathrm{l}$ of $0.1 \%$ crystal-violet solution was added and left for 10-15 mins incubation. The plate was washed and left inverted for dry. The quantitative Determination was performed by solubilizing the biofilm by adding $125 \mu \mathrm{l}$ of $30 \%$ acetic acid to each well and incubated the plate for 10-15 mins at room temperature and was transferred to another microtitre plates and reading the absorbance at $570 \mathrm{~nm}$ by ELISA plate Reader. The interpretation of biofilm production was done according to the criteria of Stepanovic et al. (2007) [19].

\begin{tabular}{ll} 
Average OD & Value Biofilm production \\
\hline$\leq \mathrm{ODc} / \mathrm{ODc}<\sim \leq 2 \times \mathrm{ODc}$ & Non/weak \\
\hline $2 \times \mathrm{ODc}<\sim \leq 4 \times$ ODc & Moderate \\
\hline$>4 \times \mathrm{ODc}$ & Strong
\end{tabular}

Optical density cut-off value $(\mathrm{ODC})=$ average $\mathrm{OD}$ of negative control $+3 x$ standard deviation negative control.

\section{Tube method}

A qualitative assessment of biofilm formation was done as described by Christensen et al (1985)[18]. The TSB glu $(10 \mathrm{~mL})$ was inoculated with a loop full of Candida albicans from overnight culture plates and incubated for 24 hours at $37^{\circ} \mathrm{C}$. The tubes was decanted and washed with PBS (pH 7.2) and dried. Then the tubes were stained by $0.1 \%$ crystal violet. Stain was removed by deionized water. Tubes were then dried in inverted position for biofilm formation. Biofilm formation was considered positive when a visible film lined the wall and bottom of the tube. Ring formation at the liquid interface was not considered biofilm formation. An experiment was repeated for three times.

\section{Congo red Agar Method (CRA)}

The Candida albicans culture was streaked on surface of Congo Red Agar and incubated at $37^{\circ} \mathrm{C}$ for $24-$ 48 hours (Freeman et al 1989) [20]. Black coloured colonies with dry crystalline consistency interpreted as positive biofilm producing strains. Red coloured colonies- interpreted as negative for biofilm production. 
All Candida albicans isolated from samples were subjected to in-vitro antifungal susceptibility test by Kirby-Bauer disc diffusion method as recommended by CLSI 2010 [21]. In this study the antifungal used were Amphotericin-B (100 Units) and fluconazole 10 (mcg) (Himedia, Mumbai India). In this study, the most preferred class of antifungal agents like polyenes and azoles was included; amphotericin-B (100 Units) and fluconazole ( $25 \mu \mathrm{g} / \mathrm{mL}$.), (HiMedia, Mumbai, India) stored in at $4^{\circ} \mathrm{C}$. Yeast inoculums were prepared by picking five distinct colonies of approximately one $\mathrm{mm}$ from each 24 hours old culture grew on Sabouraud Dextrose Agar (HiMedia, Mumbai, India) incubated at $35 \pm 2^{\circ} \mathrm{C}$. Colonies was suspended in $5 \mathrm{~mL}$ of sterile $0.85 \%$ Saline. Vortexed the resulting suspension and adjusted the turbidity to yield $1 \times 10^{6}$ $-5 \times 10^{6}$ cells $/ \mathrm{mL}$ (i.e., $0.5 \mathrm{McF}$ arland standard). A sterile cotton swab moistened with the inoculums suspension was used to apply to a $90 \mathrm{~mm}$ diameter plate containing Mueller-Hinton agar (HiMedia, Mumbai, India) supplemented with $2 \%$ glucose and $0.5 \mu \mathrm{g} / \mathrm{mL}$ methylene blue (GM-MH agar medium). The plates were allowed to dry for 5-15 minutes then after the disks were placed in the center of the agar plate. The plates were incubated for $18-24$ hours at $37 \pm 2^{\circ} \mathrm{C}$ and the slowly growing isolates will again read after 48 hours incubation. Zone sizes were measured in millimeters with Zone Scale (HiMedia, Mumbai, India); the zone diameter to the nearest point at which there was influential reduction in growth was taken into consideration. The organism was considered as resistant, intermediate or sensitive based on the standard interpretative chart according to CLSI standard (HiMedia Company, 2010).

\section{Statistical Analysis}

The data was analyzed using statistical Package for Social Sciences (SPSS) version 16.0. Mann Whitney $U$ test was carried out to calculate CFU counts, Chi Square $\left(\chi^{2}\right)$ were used for statistical analyses. The $p$ value of equal or less than 0.05 was used for statistical significance.

\section{Results}

Analysis of Candida Carriage between Diabetic cases and Healthy controls

Candida carriage expressed in CFU/ml was comparatively studies in both Case and control groups using Mann Whitney $U$ test. The result of statistical analysis of candida carriage is shown in Table 1 (Supplementary Files).

\section{Prevalence of Candida in Population}

The overall prevalence of Candida species was $31.5 \%$ whereas the prevalence of Candida albicans only was $21 \%$. The result of prevalence of Candida yeast in population is shown in Figure 3 .

\section{Comparative study of Candida albicans isolated from different populations}

Candida albicans were isolated from Diabetic population 29 followed by Barbers 15, Municipal Waste worker 6 and Healthy populations 13 respectively. The highest percentage of Candida albicans was 
isolated from Diabetic population 16 (55.17\%), followed by Barbers $15(100 \%)$, Healthy population 6 (46.15\%) and Municipal waste worker 5 (83.33\%). The Comparative study of Candida albicans isolated from different Populations is shown in Table 2 (Supplementary Files).

\section{Antifungal susceptibility pattern of Candida albicans isolates}

It was found that $76.19 \%(32 / 42)$ were Fluconazole resistant. Other antifungal agent was used to identify the susceptibility and resistant pattern of positive isolates and it was found that $50 \%(21 / 42)$ were Amphotericin Resistant. Antifungal Susceptibility Test of isolated Candida albicans is shown in Table 3 (Supplementary Files.

\section{Phospholipase and Haemolysin assay}

The Pz value of isolated Candida albicans ranged from 2 to 2.95 for all the isolates and 2.01 to 2.6 for isolates from Diabetic group and 2.09 To 2.89 for control groups. The mean Pz value in Diabetic population was 2.11 and in Control groups was 2.52.The $P_{Z}$ Values of isolates are shown in Table 4 (Supplementary Files).The $\mathrm{Hz}$ values ranged from 5 to 18 for Diabetic groups and 3.45 to 16 for Control Groups. The mean HZ value in Diabetic group was 9.28 and in Healthy controls were 7.91. The Haemolysis degrees of isolates are shown in Table 5 (Supplementary Files).

\section{Biofilm assay by microtitre plate method}

The 96-well microtitre plate method for biofilm formation was carried out. The overall biofilm producers were $69.04 \%$ and $35.96 \%$ were non biofilm producer. The strong biofilm producer was $2(4.76 \%)$, moderate biofilm producer was 27 (64.28\%) and Weak/none biofilm producer were 13 (30.96\%). Biofilm assay by microtitre plate method is shown in Table 6 (Supplementary Files).

The Biofilm formation with Antifungal Drug Resistance is shown in Figure 5.

\section{Sensitivity and Specificity of Biofilm Screening Methods}

The microtitre plate method was found to be most efficient standard method for studying biofilm formation as compared to tube method and Congo red agar method. The parameters like sensitivity, specificity, negative predictive value, positive predictive value and accuracy were calculated and are shown in Table 7 (Supplementary Files).

\section{Discussion}

The present Study was carried out at Microbiology and Molecular Biology Laboratory of Central Campus of Technology, Dharan. During the study period a total of 200 oral rinse samples were collected from 
three potentially risk populations (50 barbers, 50 municipal waste workers, 50 diabetic populations) and 50 healthy populations (Control). In the present study the overall prevalence of Candida species in sample population was 31.5\% (63/200) and the prevalence of Candida albicans was $21 \%(42 / 200)$. In this study, Candida albicans were isolated from 29 (69.04\%) male population and 13 (30.96\%) female population. Majima et al (2014) reported $17.8 \%$ oral candida carriage in male and $18.8 \%$ oral Candida carriage in female [22]. Javed et al 2009 reported greater prevalence of $C$. albicans in oral cavity of denture wearing males (74\%) than in female (23\%) with diabetic mellitus Type II disease. Loster et al (2016) reported that the infection free individual were greater in male than in female and statistically significant relationship between intensity of yeast and gender [23].

In our study the maximum frequency of organism was found in between age group of 40-50 years. The association of Candida in in different age groups was found to be statically insignificant $(p>0.05)$. Lockhart et al (1999) found the increase in oral candida carriage was reported by with age possibly due to diminished natural defenses [24]. In our study the prevalence of yeast in elderly age group was found to be least possibly due to least sample population from old age group. There was no significant association between Age and prevalence of Candida albicans.

This study reported $31.5 \%$ prevalence of oral Candida. Out of 63 positive Samples of Candida, 42 isolates were known to be Candida albicans. Around $85-90 \%$ of diabetic patients are diagnosed by Type2 Diabetes. In Diabetic patients the salivary dysfunction such as Xerostomia, tooth decay, decreased salivary flow and periodontal diseases are common (Ship 2003) [25]. Diabetic patients are more susceptible to oral Candidiasis due to increased salivary glucose, low secretion of saliva, impaired chemotaxis and defect of phagocytosis (Kadir et al 2002)[26]. Many Risk factors are associated with colonization by Candida. Poor oral hygiene, diabetic conditions, immunosuppressive therapy in Cancer disease, intake of Gutkha has shown increasing prevalence of Candida species (Guggenheimer et al 2000)[27]. Statistically significant increase in Candida carriage in terms of colony forming units were reported in patients with Diabetes mellitus than in control Groups, $p<0.05$ with CFU/ml ranging from 0 to 1700. This result is similar too many other studies that explain the higher susceptibility of Candida to colonize oral cavity of diabetic patients. The study conducted by Lamichhane et al (2015) showed significant increase in Candida CFU in Diabetic patients than in Controls [28]. Host factors which contribute to oral Candidal carriage in Diabetes are known to be Candidacidal activity of neutrophils in presence of Glucose. In diabetic patients with Candidiasis the Polymorphonuclear leucocytes (PMN) produces less free oxygen radicals exhibiting reduced phagocytosis, bactericidal activity and chemotaxis. This may confer Diabetic patients more susceptible to Oral Candida infections (Soysa et al 2005) [29]. Mohammadi F et al 2016 found 55\% Diabetic patient to carry Candida sp. in oral cavity which C. albicans was abundant species. Belazi et al (2005) isolated candida sp. from oral cavity of $64 \%$ diabetic and $40 \%$ 
control population. Many researchers conducted throughout the world have reported cases of oral candidiasis in diabetic patients commonly known as oral thrush [3].

Pathogenic microorganisms are capable of acquiring iron for survival and establish infection in host that addresses its pathogenicity. Since there is little iron human body, the most microorganisms derive iron from hemoglobin. So to destroy hemoglobin, they secrete enzyme like haemolysin (Malcok et al 2009) [12].The mean hemolytic degree of Candida albicans in human blood with Glucose (SDBwG) was 58.68\% and in human blood without glucose (SDBwoG) was $48.74 \%$. We reported that Candida albicans exhibited higher hemolytic degree. Malcok et al (2009) reported in his study that most species of Candida exhibited hemolytic activity in $3 \%$ glucose enriched medium and hence suggested the parallel combination of Diabetic condition with pathogenesis of Candida albicans [12].In-vitro analysis of hemolytic degree of Candida albicans in presence and absence of glucose level strongly explains the increased blood sugar level increases the Hemolysin activity of Candida albicans. This study suggests the pathogenic role of Candida albicans in Diabetic mellitus and supports the concept of susceptibility of Candida colonization and Candidiasis in Diabetic patients. The Haemolysin Index of Candida albicans isolated from Diabetic patients was found higher than in Control population.

The Pz value of isolated Candida albicans ranged from 2.01 to 2.6 for isolates from Diabetic group and 2.09 To 2.89 for control groups. The mean Pz value in Diabetic population was 2.11 and in Control groups were 2.52. There was no significant difference in phospholipase activity in Diabetic and control groups ( $p>0.05)$. Tsang et al (2007) reported no significant difference among phospholipase activity among Candida albicans isolated from Diabetic and Control groups. Tsang et al (2007) reported higher proteinase and Haemolytic activity from Diabetic patients with no significant difference in phospholipase activity from test and control groups [29]. Sachin et al (2012) reported 94.8\% C. albicans exhibiting higher haemolysin activity and $92.3 \%$ exhibiting phospholipase activity [30]. In our study all strains of $C$. albicans exhibited haemolysin activity and only $68 \%$ C. albicans exhibited phospholipase activity. In our study there was significant difference in Hemolysin activity among Diabetic and control groups $(p=0.049)$.

Candida has known to be opportunistic pathogen under immunosuppression and tobacco chewing conditions [6, 7]. It is suggested that individuals chewing Gutkha are susceptible to oral Candida infections than non-chewers [8]. Chewing Tobacco that includes Gutkha, Betel quid (BQ) which is common habit in South Asian nations like in India, Pakistan, Bangladesh, Sri lanka and Nepal [31]. Betel quid is mixture of areca-nut, lime enveloped in piper betel leaf whereas Gutkha is found in Sachet [32]. The possible Explanation for greater Candida colonization in Gutkha consumers could be due to the presence of nicotine and hydrocarbons such as polycyclic aromatic hydrocarbons acting as nutrient for Oral yeast facilitating its growth [7.8]. In our study, higher prevalence of Candida carriage was found in Gutkha chewers. The Candida colonization and Gutkha consumption was statistically significant 
,p<0.05.Similar study by Abduljabbar et al (2017) reported a significant difference in Oral Candida carriage in Gutkha chewer and Betel Quid chewer suggesting the susceptibility to oral candidiasis in those risk groups than in control groups[8]. However Javed et al (2014) showed no significant difference in oral Candida colonization among Gutkha and Betel quid chewer [6]. This contradiction in our study could be due to fact that Oral rinse sample were obtained for complete enumeration of Candida from oral cavity. The prevalence of oral Candida carriage in healthy population having good oral hygiene was low as compared to others. The healthy populations included individuals without physical complications and diseases, proper BMI, and no smoking, chewing and Drinking Habit. The lower frequency of Candida carriage in healthy might be due to maintenance good oral hygiene and practice. We can at least explain that being a part of normal flora the colonization of Candida is associated even with poor oral hygiene.

All isolated strains of Candida albicans were tested for antifungal susceptibility testing by using Kirby Bauer disk diffusion method $76.19 \%$ were found to be Resistant to Fluconazole and $50 \%$ were found to be resistant to Amphotericin B. It was observed that emergence of drug resistance in Candida albicans is a major global issue which persuade for new search of therapeutic agents for eradicating the infections. The emerging Drug Resistance is has become global burden for treating the infections. Candida infection has been increasing in immunocompromised, over aged, patients receiving cancer chemotherapy and organ transplantation.

Biofilm is the surface-attached microbial community that contributes virulence factor. Biofilm helps Candida to escape host defense and develop resistance to antimicrobial agents. Over last two decades the burden of Candida infection throughout the world has been rising with drug resistance. Diabetes mellitus patients, immuno-compromised patients like HIV patients, antibiotic users, IV users, Gutkha consumers etc. are at risk to Candida infection. Biofilm of Candida is made up of layers of cells embedded in matrix of extracellular polymeric materials (Khatri et al 2015) [33].

In our study the strong, moderate and weak biofilm producers by microtitre plate method were $4.7 \%$, $64.2 \%$ and $30.9 \%$ respectively. The strong, moderate and weak biofilm producers by TM method were $4.7 \%, 38 \%$ and $57.1 \%$ respectively. The strong, moderate and weak biofilm producers by CRA method were $2.3 \%, 35.7 \%$ and $61.9 \%$ respectively. Number of false positive and false negative was reported in the comparison. It was difficult to discriminate strong, moderate and weak biofilm producers in Tube Method and Congo Red Agar method due to phenotypic variations. Sensitivity and specificity of TM was found to be $71.4 \%$ and $62.8 \%$ respectively with accuracy of $64.2 \%$. For Congo red agar method the sensitivity and specificity was found to be $27.7 \%$ and $16.6 \%$ with accuracy of $21.4 \%$. The statistical analysis of screening were similar even in our findings which supports different other similar findings done before. Hassan et al (2011) concluded Microtitre method as gold standard technique for screening biofilm as 
compared to Tube method and Congo Red Agar [34]. Even in our study The Microtitre method was considered efficient method for quantitative screening of biofilm in comparison to TM and CRA methods.

In our study $4.7 \%$ of isolated Candida albicans were strong biofilm producers, $64.2 \%$ were moderate and $30.9 \%$ were weak or non-biofilm producer. In overall the biofilm forming Candida albicans were $69 \%$ and non-biofilm producers were $30.9 \%$. In our study the maximum frequency of Candida albicans were biofilm producers and even maximum biofilm producing Candida albicans were resistant to antifungal drugs. This association may explain the ability of biofilm production with drug resistance in the organism exhibiting its strong virulence factor and making failure of antifungal drugs. Mohammadi et al (2016) reported that biofilm formation and overgrowth of Candida is found more in diabetic patients [35]. Similar to it even in our study the antifungal resistance pattern of Candida albicans showed highest resistance to fluconazole whereas Amphotericin B also showed good sensitivity.

The biofilm formation and Fluconazole resistance was statistically significant $p<0.05$, whereas the biofilm formation and Amphotericin B drug resistance was not significant. Amphotericin B showed a greater Sensitivity towards Candida albicans. Weak biofilm producing Candida albicans isolates showing resistance towards Amphotericin B could be due to efflux pump other than biofilm. Most studies suggest that Amphotericin B displays a good activity against Candida biofilms. According to study conducted by Mahmoudabadi, Zarrin et al (2014) the $100 \%$ isolates of Candida albicans were biofilm producers where $65 \%$ showed Sensitivity towards Amphotericin B and only $26.7 \%$ Sensitivity towards Fluconazole [36]. Similar findings were observed even in our study.

In Nepal, improper use of antibiotics drugs without doctor's prescription, haphazard selling and buying of antibiotic from local pharmacy and unnecessary use of drugs have been responsible for drug resistance to microorganisms. This uncontrolled use of drug has led to antifungal drug resistance in strains of microorganisms. Indiscriminate use of drugs in our community is a major cause for emergence of drug resistance. The proper availability of microbiological investigation and information would be necessary in search of appropriate antimicrobial agents to fight with drug resistance. Since for many years Fluconazole has been used for treatment of oral candidiasis but the emergence of drug resistance renders the search of alternative antimicrobial therapy.

The good oral hygiene and the reduced prevalence of Candida carriage was statistically significant, p $<0.05$. In our study the total Candida carriage among healthy population was only $26 \%$. Majima et al (2014) found $18.3 \%$ oral Candida carriage among dental students and suggested that good oral hygiene responsible for elimination of Candida [22]. Therefore with overall analysis in potential risk groups and in 
Healthy population, the oral Candida colonization shows a significant association with oral hygiene and serum glucose.

Higher prevalence of Candida in diabetic population and in poor oral condition alarms the oral health warnings. The absence of Candida in oral cavity may be due to maintenances of good oral health. Thus, oral carriage of Candida serves for health issues as oral candidiasis requires initial colonization by Candida.

\section{Declarations}

Source of Support: None

Acknowledgements: The authors want to thank participants and Central Campus of Technology, Tribhuvan University.

Conflict of interest: Authors declare no Conflict of interest.

Ethics approval and consent of participation: The ethical approval was obtained by Nepal Health Research Council, Nepal. Written Informed Consents were obtained from the participants before starting this study.

\section{References}

1. Lamont RJ, Burne RA, Lantz MS, Leblanc DJ. Fungi and fungal infections of the oral cavity. American Society of Microbiology. 2006; pp. 333-348.

2. Kumamoto CA. "Candida biofilms". Current Opinion in Microbiology. 2002;5(6): 60811. doi:1016/s1369-5274(02)00371-5. PMID12457706.3

3. Chung LM, Liang JA, Lin CL, Sun LM, Kao CH. Cancer risk in patients with candidiasis: a nationwide population-based cohort study. Oncotarget. 2017;8(38):63562-63573

4. Obradovic R R., L. G. Kesic, A. N. Pejcic, M. S. Petrovic, N. D. Zivkovic and D. M. zivkovic. "Diabetes mellitus and oral candidiasis." Acta Stomatologica Naissi. 2011;27(63): 1025-1034.

5. Mehrotra R, Hullmann M, Smeets R, Reichert TE, Driemel O. Oral cytology revisited. J Oral Pathol Med. 2009;38:161-166. Crossref.

6. Javed F, Tenenbaum HC, Nogueira-Filho G, Nooh N, Taiyeb Ali TB, Samaranayake LP. Oral Candida carriage and species prevalence amongst habitual gutka-chewers and non-chewers. Int Wound J. 2014;11:79-84.

7. Hsia CC, Sun TT, Wang YY, Anderson LM, Armstrong D, Good RA. Enhancement of formation of the esophageal carcinogen benzylmethylnitrosamine from its precursors by Candida albicans. Proc Natl Acad Sci USA. 1981;78:1878-81. 
8. Abduljabbar T, Hussain M, Adnan T, Vohra F and Javed F. "Comparison of oral Candida species prevalence and carriage among gutka-chewers and betel-quid chewers." J Pak Med Assoc. 2017;67: 350-354.

9. Javed, F., L. Klingspor, U. Sundin, M. Altamash, B. Klinge and P.-E. Engström. "Periodontal conditions, oral Candida albicans and salivary proteins in type 2 diabetic subjects with emphasis on gender." BMC Oral Health. 2009;9(1): 12.

10. Silva S, Negri M, Henriques M, Oliveira R., Williams D W \& Azeredo J. Candida glabrata, Candida parapsilosis and Candida tropicalis: biology, epidemiology, pathogenicity and antifungal resistance. FEMS Microbiol Rev. 2011;36, 288-305.

11. Ozkan S, Kaynak, F, Kalkanci, A, Abbasoglu U. and Kustimur S. Slime production and proteinase activity of Candida species isolated from blood samples and the comparison of these activities with minimum inhibitory concentration values of antifungal agents. Mem Inst Oswaldo Cruz. 2005;100, 319-323.

12. Malcok HK, E. Aktas A, Ayyildiz, N Yigit and H Yazgi. "Hemolytic activities of the Candida species in liquid medium" The Eurasian journal of medicine. 2009;41(2): 95.

13. Luo G, Samaranayake LP, Yau JY. Candida species exhibit differential in vitro hemolytic activities. J Clin Microbiol. 2001;39(8):2971-4. doi: 10.1128/JCM.39.8.2971-2974.2001.

14. Lamichhane RS, Boaz K, Natarajan S, Shrestha M. Assessment of Candidal carriage in patients with Type II Diabetes Mellitus. Journal of Pathology of Nepal. 2015;5, 733-738.

15. Belazi, M., A. Velegraki, A. Fleva, I. Gidarakou, L. Papanaum, D. Baka, N. Daniilidou and D. Karamitsos. "Candidal overgrowth in diabetic patients: potential predisposing factors." Mycoses. 2005;48(3): 192-196.

16. Beheshti, F., A. G. Smith and G. W. Krause. "Germ tube and chlamydospore formation by Candida albicans on a new medium." Journal of clinical microbiology. 1975;2(4): 345-348.

17. Samaranayake LP, Raeside JM and MacFarlane TW. Factors affecting the phospholipase activity of Candida species in vitro. Sabouraudia. 1984;22, 201-207.

18. Christensen GD, Simpson WA, Younger JJ, Baddour LM, Barrett FF, Melton DM, Beachey EH. Adherence of coagulase-negative Staphylococci to plastic tissue culture plates: a quantitative model for the adherence of staphylococci to medical devices. J Clin Microbiol. 1985;22(6):996-1006.

19. Stepanovic, S., D. Vukovic, V. Hola, G. D. Bonaventura, S. Djukić, I. Ćirković and F. Ruzicka. "Quantification of biofilm in microtiter plates: overview of testing conditions and practical recommendations for assessment of biofilm production by staphylococci." Apmis. 2007;115(8): 891899.

20. Freeman D J, Falkiner F R, Keane $C$ T. New method for detecting slime production by coagulase negative Staphylococci. J Clin Pathol. 1989; 42(8):872-4.

21. Clinical and Laboratory Standards Institute. Performance standards for antifungal disk diffusion susceptibility testing of non-dermatophyte filamentous fungi; Informational supplement-First edition. 
2010; CLSI document M51-A. Villanova, PA: Clinical and Laboratory Standards Institute; Back to cited text no. 31.

22. Majima T, S. Ito-Kuwa, R. Nagatomi and K. Nakamura. "Study of the oral carriage of Candida sp. in dental students and staff-Identification of Candida sp. and background survey." Oral Science International. 2014;11(1): 30-34.

23. Loster JE, Wieczorek A, Loster BW. Correlation between age and gender in Candida species infection of complete denture wearers a retrospective analysis. Clinical interventions in aging. 2016;11:17071714.

24. Lockhart S.R, Vargas K, Swails-Wenger J, Enger L, Soll DR. Natural defenses against Candida colonization Breakdown in oral cavities of the ederly. Journal of Dental Research. 1999;78(4).

25. Ship JA. Diabetes and oral health an overview. J Am Dent Asso. 2003;134:4S-10.

26. Kadir T, Pisiriciler R, Akyüz S, Yarat A, Emekli N, Ipbüker A. Mycological and cytological examination of oral candidal carriage in diabetic patients and nondiabetic control subjects: thorough analysis of local aetiologic and systemic factors. J Oral Rehabil. 2002;29(5):452-7.

27. Guggenheimer J, Moore PA, Rossie K, Myers D, Mongelluzzo MB, Block HM. Insulindependent diabetes mellitus and oral soft tissue pathologies. II. Prevalence and characteristics of Candida and candidal lesions. Oral Surg Oral Med Oral Pathol Oral Radiol Endodontol. 2000;89(5):570-6.

28. Soysa NS, Samaranayake LP and Ellepola ANB. Diabetes mellitus as a contributory factor in oral candidosis. Diabetes Medicine. 2005;23:455-459.

29. Tsang, C., F. Chu, W. Leung, L. Jin, L. Samaranayake and S. Siu. Phospholipase, proteinase and haemolytic activities of Candida albicans isolated from oral cavities of patients with type 2 diabetes mellitus.Journal of medical microbiology. 2007;56(10): 1393-1398.

30. Sachin CD, Ruchi K, Santosh S. In vitro evaluation of proteinase, phospholipase and haemolysin activities of Candida species isolated from clinical specimens. International journal of medicine and biomedical research. 2012;1(2).

31. Javed F, Chotai M, Mehmood A, Almas K. Oral mucosal disorders associated with habitual gutka usage: a review. Oral Surg Oral Med Oral Pathol Oral Radiol Endod. 2010;109:857-64.

32. Javed F, Yakob M, Ahmed HB, Al-Hezaimi K, Samaranayake LP. Comparison of oral Candida species prevalence and carriage among gutka-chewers and betel-quid chewers 353 Candida carriage among individuals chewing betel-quid with and without tobacco. Oral Surg Oral Med Oral Pathol Oral Radiol. 2013;116:427-32.

33. Khatri S, M. Sumana RP Mahale and A. Kishore. "Analysing three different screening methods for biofilm formation in clinical isolates of Candida." Journal of Evolution of Medical and Dental Sciences. 2015;4(83): 14515-14524.

34. Hassan A, Usman J, Kaleem F, Omai M, Khalid A, Iqbal M. Evaluation of different detection methods of biofilm formation in the clinical isolates. Brazilian journal of infectious Diseases. 2011;15(4); 305311. 
35. Mohammadi F, Javaheri MR, Nekoeian S, Dehghan P. Identification of Candida species in the oral cavity of diabetic patients. Curr Med Mycol. 2016;2(2):1-7.

36. Mahmoudabadi, A. Z., M. Zarrin and N. Kiasat. "Biofilm formation and susceptibility to amphotericin B and fluconazole in Candida albicans." Jundishapur journal of microbiology. 2014;7(7).

\section{Tables}

Due to technical limitations, tables $1-7$ are only available as a download in the supplemental files section.

\section{Figures}

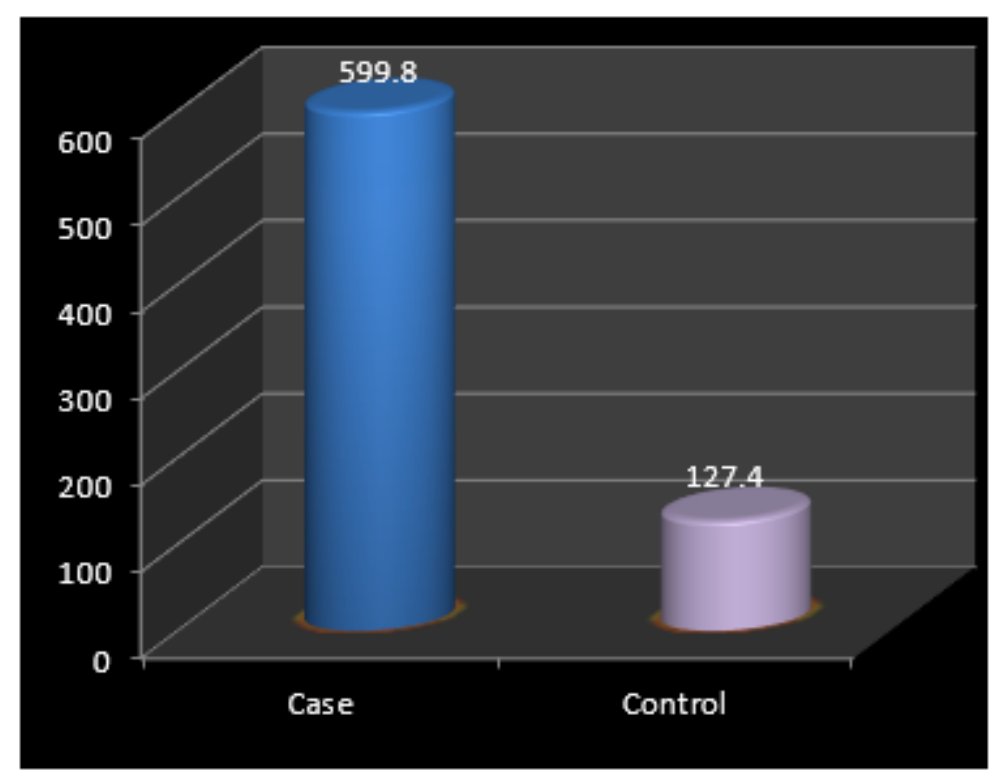

\section{Figure 1}

Distribution of colony forming units (CFUs) among Diabetic cases and Healthy control.

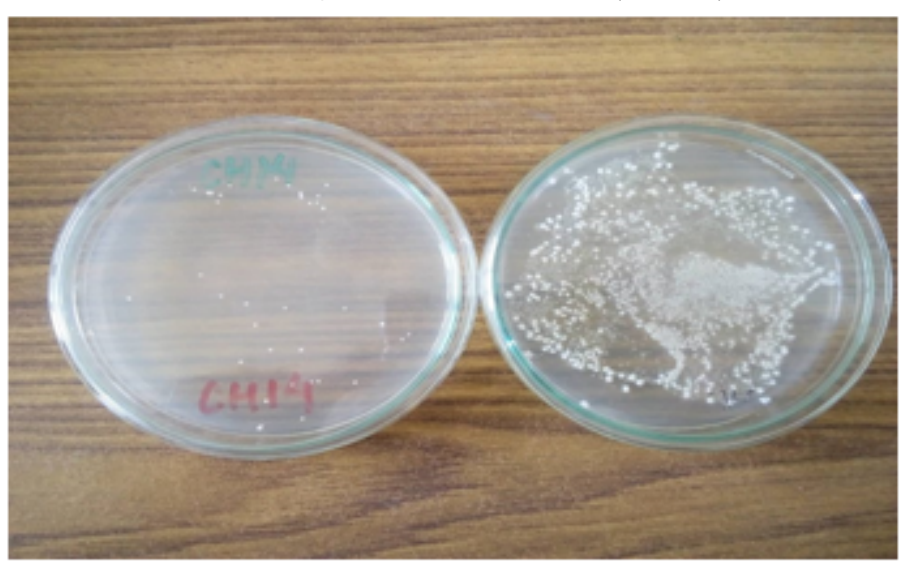

Figure 2 
Candida Carriage in CFU from Healthy (Left) and Diabetic case (Right)

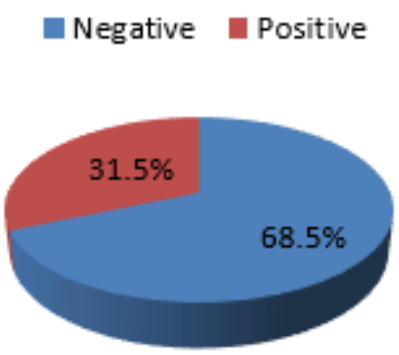

\section{Figure 3}

Prevalence of Candida in Population

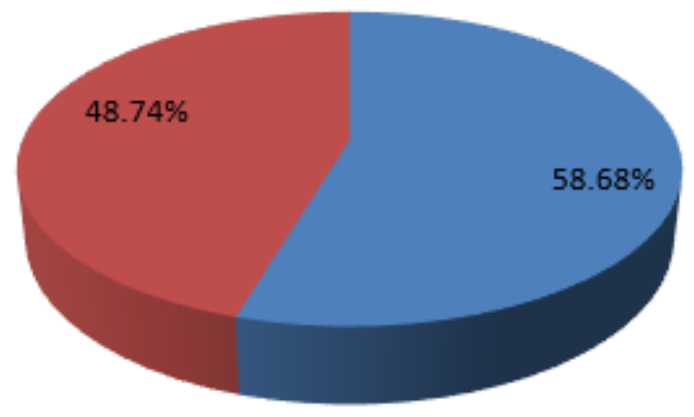

$$
\begin{aligned}
& \text { SDBwGlu } \\
& \text { SDBwoGlu }
\end{aligned}
$$

\section{Figure 4}

Hemolysis Degree exhibited by isolated Candida albicans in SDB with and without Glucose 

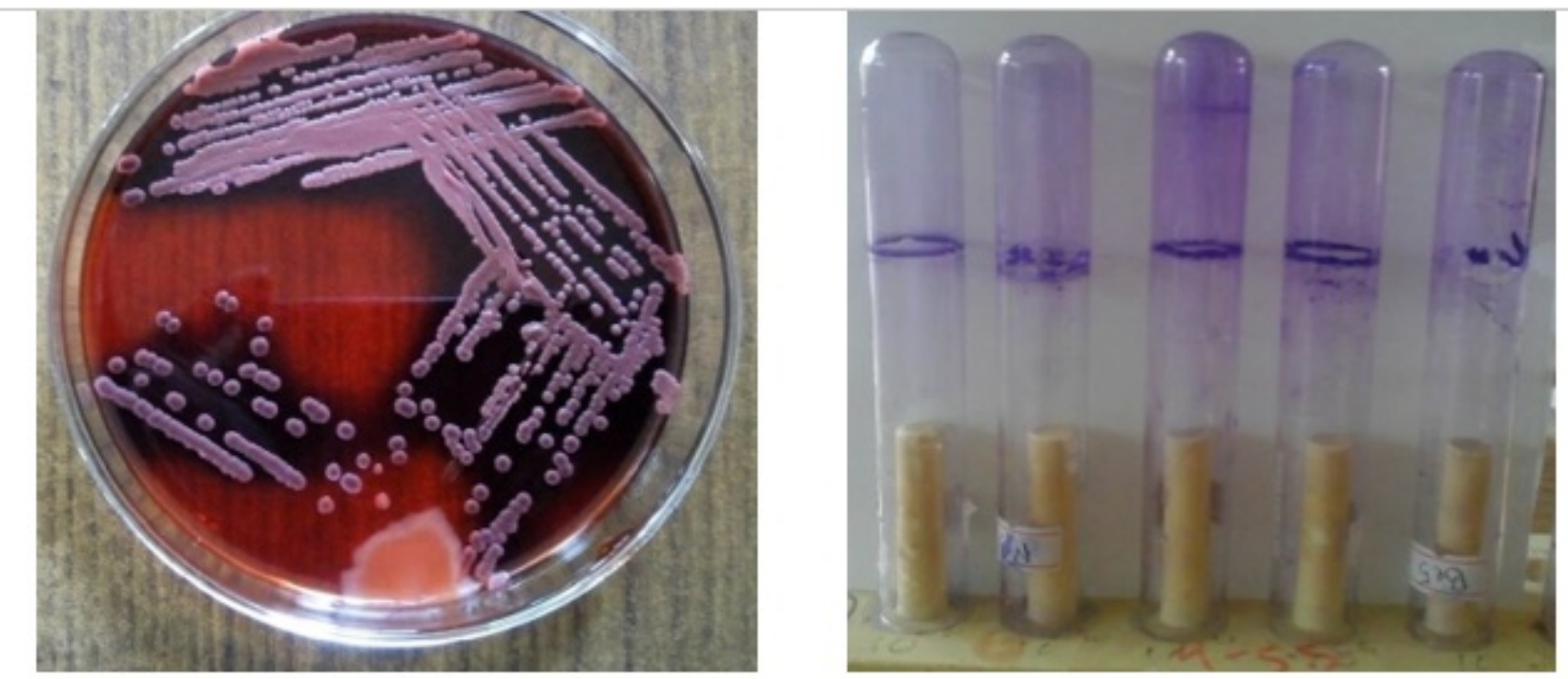

A

B

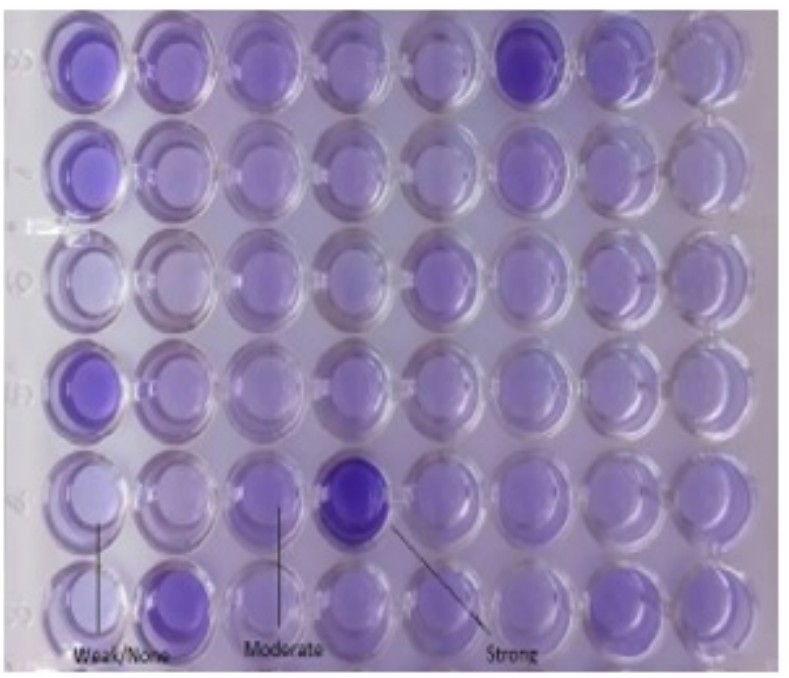

$\mathrm{C}$

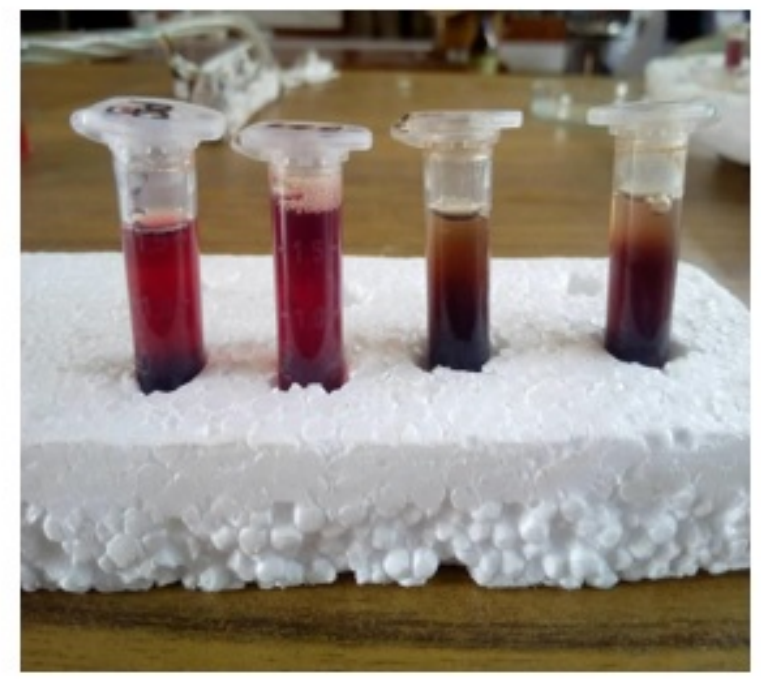

D

Figure 4: Biofilm formation by different Techniques and Haemolysis Degree Biofilm formation by Candida albicans on Congo Red agar characterized by black colony (A).Biofilm formation by Candida albicans on Tube (B). Biofilm formation by Candida albicans on Microtitre plate(C).Hemolysis Degree exhibited by Candida albicans on SDB with Glucose (Right) and SDB without Glucose (Left) (D).

\section{Figure 5}

Biofilm formation by different Techniques and Haemolysis Degree Biofilm formation by Candida albicans on Congo Red agar characterized by black colony (A).Biofilm formation by Candida albicans on Tube (B). Biofilm formation by Candida albicans on Microtitre plate $(C)$. Hemolysis Degree exhibited by Candida albicans on SDB with Glucose (Right) and SDB without Glucose (Left) (D).

\section{Supplementary Files}


This is a list of supplementary files associated with this preprint. Click to download.

- Table7.jpg

- Table4.jpg

- Table5.jpg

- Table3.jpg

- Table6.jpg

- Table1.jpg

- AdditionalFigure.png

- Table2.jpg 